\title{
Determination of target depth in a turbid medium with polarization-dependent transmitted signals
}

\author{
Chia-Wei Sun, Kuei-Chao Liu, Yih-Ming Wang, Hsiang-Hsu Wang, Yean-Woei Kiang, Hua-Kuang Liu, \\ and C. C. Yang \\ Graduate Institute of Electro-Optical Engineering, Graduate Institute of Communication Engineering and \\ Department of Electrical Engineering, National Taiwan University, No. 1, Section 4, Roosevelt Road, Taipei, Taiwan
}

Received December 27, 2002; revised manuscript received July 7, 2003; accepted July 18, 2003

\begin{abstract}
We demonstrate a novel method for target depth determination in a turbid medium with experiments and Monte Carlo simulations. This method relies on the strong dependence of transmitted co-polarized intensity on target depth. Such a dependence originates from the inclusion of certain diffuse photons in the copolarized intensity. A target of stronger scattering located closer to the transmitter results in stronger photon divergence and hence weaker co-polarized intensity at the receiver of a finite aperture. On the other hand, the degree of polarization (DOP) carries only information about ballistic and snake photons. It is weakly dependent on the target depth. The DOP data can be used as a reference of absolute scattering strength in the turbid system. Our experimental and Monte Carlo simulation results show the feasibility of the proposed target depth determination method. Meanwhile, it is shown that an appropriate time-gating process could help in improving the accuracy of target depth. In addition, the results show that the proposed method has quite large applicable ranges of scattering coefficient and absorption coefficient. (c) 2003 Optical Society of America

OCIS codes: 290.7050, 170.7050.
\end{abstract}

\section{INTRODUCTION}

Target identification in a turbid medium is an important research topic that can find applications in numerous areas, most notably in atmospheric optics and underwater acoustics and, more recently, in biomedical optics. In many situations, signals transmitted through turbid media are used for imaging purposes. However, multiply scattered photons carry less information about the location of a target embedded in a turbid medium because multiple scattering results in randomizations of optical propagation direction, phase, and polarization state of incident light. In this situation, such an imaging method may include the uses of time-gating ${ }^{1-3}$ and polarization-discrimination ${ }^{4-6}$ procedures to extract coherent and/or quasi-coherent photons for constructing the image of a target. Typically, time gating and polarization discrimination result in relatively weak signals; however, they lead to high-quality images. Based on these methods, one can usually obtain satisfactory two-dimensional images.

However, the determination of target depth in a turbid medium is usually quite difficult with transillumination measurements. Although scanning from different angles can provide information about target depth, such a procedure needs extensive measurement of light intensity around the target and makes the measurement more costly and time-consuming. Besides the angle-scanning method, inverse image reconstruction methods based on scattered diffuse photons are also available for three- -dimensional imaging. ${ }^{7-9}$ However, such a method requires complicated algorithms and long computation times for retrieving target images. Meanwhile, imaging based on diffuse photons usually has relatively poor resolution.

In this paper, we further explore a novel method for the determination of target depth in a turbid medium based on transillumination measurement with a polarizationdiscrimination procedure. ${ }^{10}$ With the measurement of the transmitted co-polarized intensity, a target depth indicator can be calculated. In this target depth determination process, the data of degree of polarization (DOP) can be used as the reference in identifying the overall scattering strength of a system. Besides the experimental demonstration, Monte Carlo simulation algorithms have been developed to show quite consistent results. In this paper, besides more detailed descriptions of research approaches and results, we focus on the applicability of the proposed target depth determination method when the target and the background media have strong absorption. Experimental and simulation results show that this method is still applicable in media of significant absorption (absorption coefficient up to $0.3 \mathrm{~cm}^{-1}$ ). Meanwhile, we show experimentally that the target depth accuracy can be improved with appropriate time gating of the time-dependent co-polarized intensity distribution.

This paper is organized as follows: In Section 2, the problem geometry and the experimental procedures are described. The experimental results are shown in Section 3. Then, in Section 4, the Monte Carlo method is 
briefly introduced, and the simulation results are discussed. Finally, conclusions are drawn in Section 5.

\section{PROBLEM GEOMETRY AND EXPERIMENTAL PROCEDURES}

The problem geometry is shown in Fig. 1. We assumed a slab of turbid medium of thickness $h$, in which a thinner target layer of thickness $t$ with a sharp boundary at the origin of the transverse coordinate $y$ was embedded. The optical scattering properties of the target medium were different from those of the background turbid medium. Assume that a light source (the transmitter) radiated incident light wave into the turbid medium at wavelength $800 \mathrm{~nm}$. Transmitted signals were recorded with a photodetector (the receiver) of a finite aperture, which was placed at a distance of $l$ from the turbid medium boundary and was aligned with the incident direction of signals. With the A-scan mode, the turbid medium was scanned along the $y$ direction over a range when the center location (the depth) of the target layer, i.e., $x$, varied. The turbid background medium was assumed to be a polystyrene microsphere suspension, and the target was a solidified microsphere suspension of a different concentration.

The experimental setup is shown in Fig. 2. Here, a Verdi-laser-pumped mode-locked Ti:sapphire laser was used to provide $76-\mathrm{MHz}, \sim 100-\mathrm{fs}$ laser pulses at $800 \mathrm{~nm}$. The laser beam was split into two branches, one for triggering the streak camera used and another for propagating through a sample. The transmitted signal of the sample was directed to the streak camera with a fiber bundle $(l=10 \mathrm{~cm}$ away from the turbid medium with an aperture of $1 \mathrm{~mm}$ in diameter). The geometry of the acrylic container of microsphere suspension was $5 \mathrm{~cm}$ $\times 5 \mathrm{~cm} \times 2.5 \mathrm{~cm} \quad$ (signal transmission length $h$ $=2.5 \mathrm{~cm}$ ). Two polarizers, one before and another after the container, were used to control the input and output

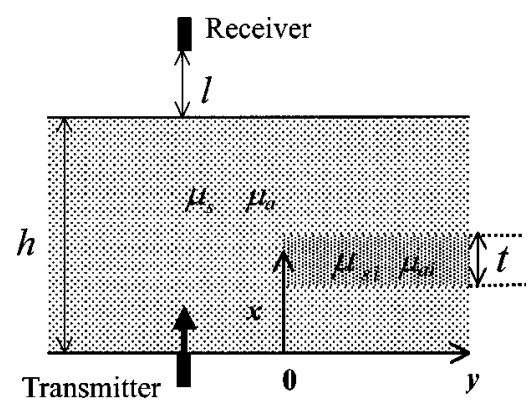

Fig. 1. Geometry of the turbid medium system under study.

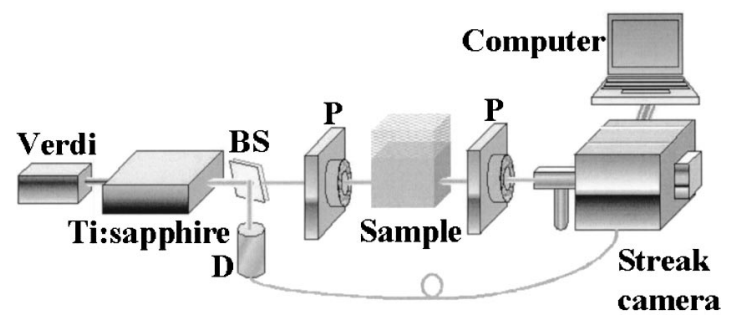

Fig. 2. Experimental setup for target depth determination. $\mathrm{P}$, polarizer; D, detector; BS, beam splitter. polarization for polarization discrimination. The A-scan mode was used in measurements; i.e., the fiber bundle end was always aligned with the incident laser beam while the samples were moved with a translation stage for image scanning. The one-dimensional scanning step size was $1 \mathrm{~mm}$, and the scanning length was $1.8 \mathrm{~cm}$. In sample preparation, white polystyrene latex was diluted with water to serve as the background turbid medium. The average diameter of the polystyrene microspheres (refractive index 1.565) was $1.8 \mu \mathrm{m}$. The volume concentration of the diluted polystyrene suspension was $0.067 \%$. The inhomogeneous target was jellied $0.134 \%$ polystyrene suspension with thickness $t=4 \mathrm{~mm}$. It had a sharp boundary at the origin of the transverse coordinate $(y=0)$.

\section{EXPERIMENTAL RESULTS}

Figure 3(a) shows the experimental results of the transmitted co-polarized intensities as functions of the transverse scanning position $y$ for four different target depths $x$. These intensities were obtained by integrating over the whole range of the time-resolved co-polarized intensity profiles. The curves show the results of transverse scanning across the boundary of the target at $y=0$. Abrupt changes of transmitted co-polarized intensity across the target boundary can be clearly seen. Although the transmitted intensity on the target-free side is almost constant with varying target depth, that on the target side increases significantly with target depth. Such variations can be explained as follows. When the target (with a higher scattering coefficient) is closer to the transmitter (smaller $x$ ), the scattered photons travel a longer distance after the influence of the target of stronger scattering. In this situation, photons (especially the diffuse photons) of random propagation directions evolve into a weaker intensity at the receiver of a finite aperture, particularly in the co-polarized component. With this dependence of transmitted intensity, the depth information can be extracted.

DOP is defined as

$$
\mathrm{DOP}=\frac{I_{\mathrm{co}}-I_{\mathrm{cr}}}{I_{\mathrm{co}}+I_{\mathrm{cr}}} .
$$

Here, $I_{\text {co }}$ and $I_{\text {cr }}$ are the co-polarized and cross-polarized intensities, respectively. Figure 4 shows the DOP distribution under the same conditions as those of Fig. 3(a). Again, these data were obtained by integrating over the whole range of the time-resolved intensity profiles. The DOP values drop from approximately 0.48 to approximately 0.27 when we scanned across the target boundary. The almost constant DOP value on the target side with varying target depth represents a major difference from the results of co-polarized intensity. Such a difference is due to the fact that the DOP value, controlled by ballistic and snake photons, is mainly determined by the total length of nearly straight photon paths, which is less influenced by the target depth. On the other hand, diffuse photons contribute to the co-polarized intensity, resulting in its dependence on target depth. Note that as the target depth $x$ increases, the DOP image around the target 


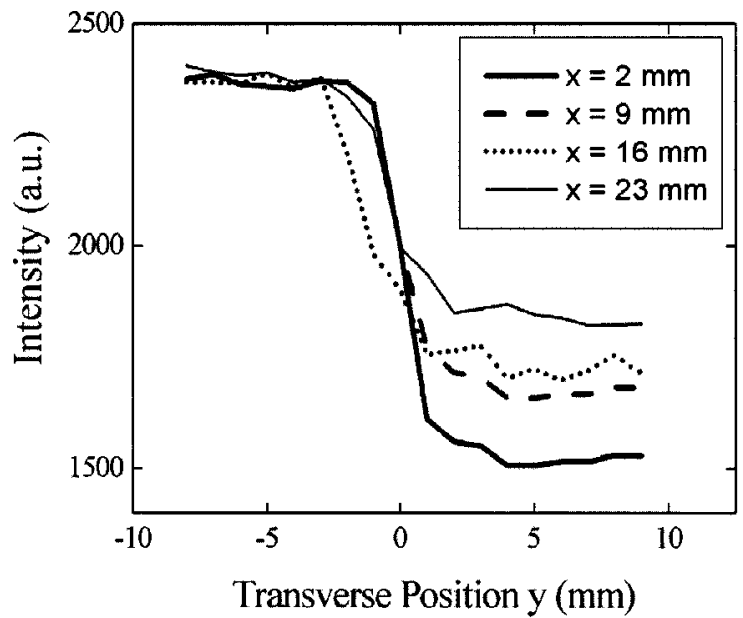

(a)

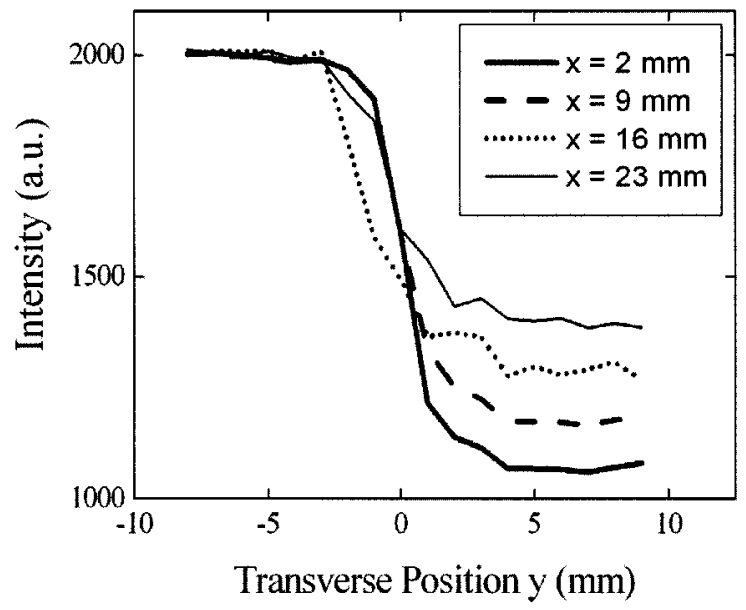

(b)

Fig. 3. Transmitted co-polarized intensity distribution of four target depths obtained from the experiments in the cases (a) without time gating and (b) with time gating.

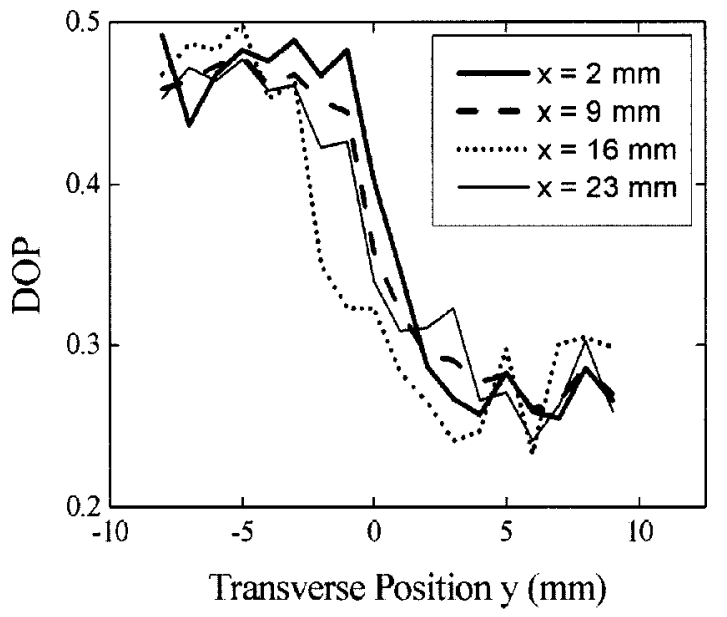

Fig. 4. DOP distribution of four target depths obtained from the experiments in the case without time gating.

edge at $y=0$ becomes blurred. In other words, the slope of the curve around $y=0$ becomes shallower as $x$ increases. The same trend can also be seen in Fig. 3(a). This trend is consistent with the so-called "shower cur- tain" effect, ${ }^{11}$ which refers to the fact that the blurring effect caused by a shower curtain is stronger when the observer is closer to the curtain.

The weak and strong dependencies of the DOP and the co-polarized intensity, respectively, on the target depth suggest a means for extracting the depth information from the co-polarized intensity data while using the DOP for lateral two-dimensional images. For this purpose, we define a depth indicator $P(x)$ as

$$
P(x)=\frac{I_{\mathrm{co}}^{\prime}(x)-I_{\mathrm{co}}^{\prime}(h-x)}{I_{\mathrm{co}}^{\prime}(x)+I_{\mathrm{co}}^{\prime}(h-x)} .
$$

Here, $I_{\text {co }}^{\prime}$ is the co-polarized intensity averaged over the scanning range $y>0$ for the target depth $x$. To evaluate $P(x)$, we need to make a two-way measurement by exchanging the positions of the transmitter and the receiver. Curve a in Fig. 5 shows the variation of the depth indicator $P(x)$ with the target depth $x$. A negative (positive) $P$ value means that the target is closer to the transmitter (receiver) in the case in which the scattering coefficient of the target is larger than that of the background turbid medium. An almost linear behavior of $P(x)$ can be seen, implying the feasibility of depth determination with the two-way measurement of the transmitted co-polarized intensity. The error bars in Fig. 5 were calibrated from the spatial fluctuations of the co-polarized intensity on the target side.

As mentioned above, the different dependencies on target depth between the co-polarized intensity and the DOP are attributed to the inclusion of certain diffuse photons in the measured co-polarized intensity. The inclusion of weakly diffusive photons is the key to depth determination. However, the inclusion of strongly diffusive photons would reduce the accuracy of depth determination. It is expected that with an appropriate time-gating procedure, depth determination can be more accurate. The time-gated results were obtained by integrating the timeresolved intensity profiles with duration of $50 \mathrm{ps}$ from the leading edges of the profiles. Figure 3(b) shows the timegated distributions of the co-polarized intensity, corresponding to the results in Fig. 3(a). The time-gating process excludes the highly diffusive photons, which correspond to the tail part of the time-resolved profile beyond the half-maximum intensity. Similar to the results

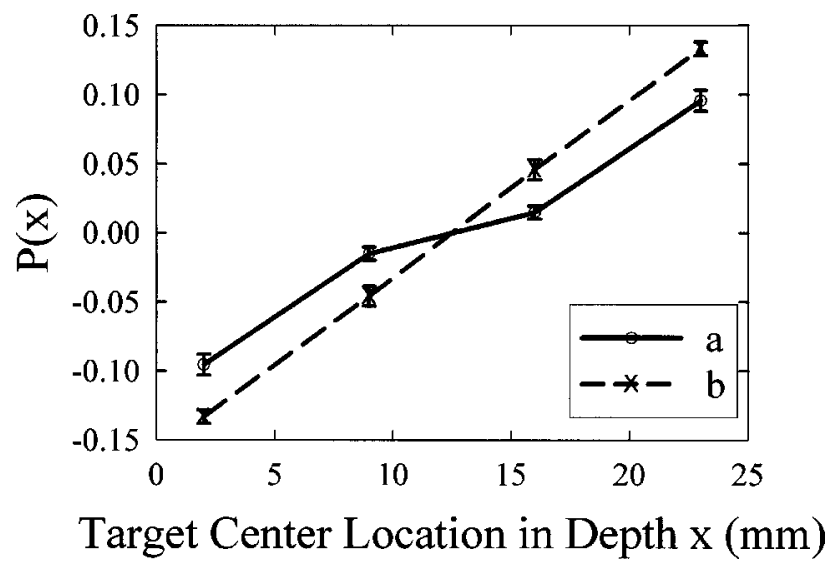

Fig. 5. Depth indicator $P(x)$ versus target depth $x$ in the cases without time gating (curve a) and with time gating (curve b). 


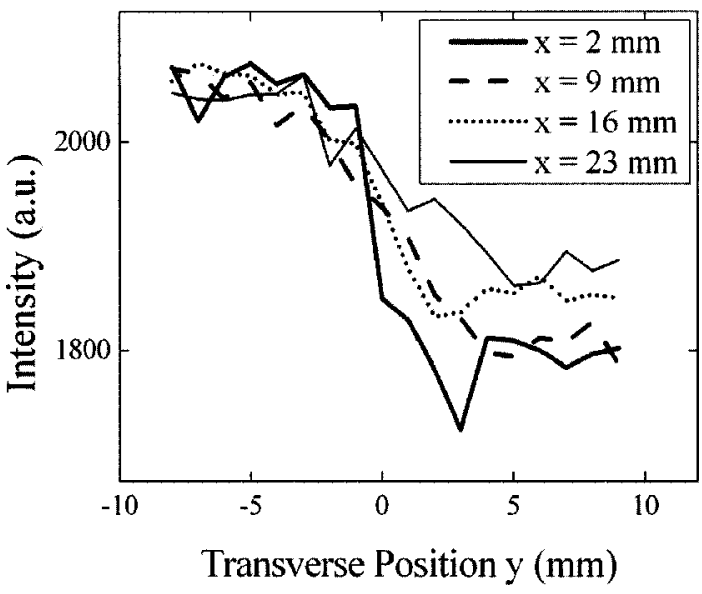

(a)

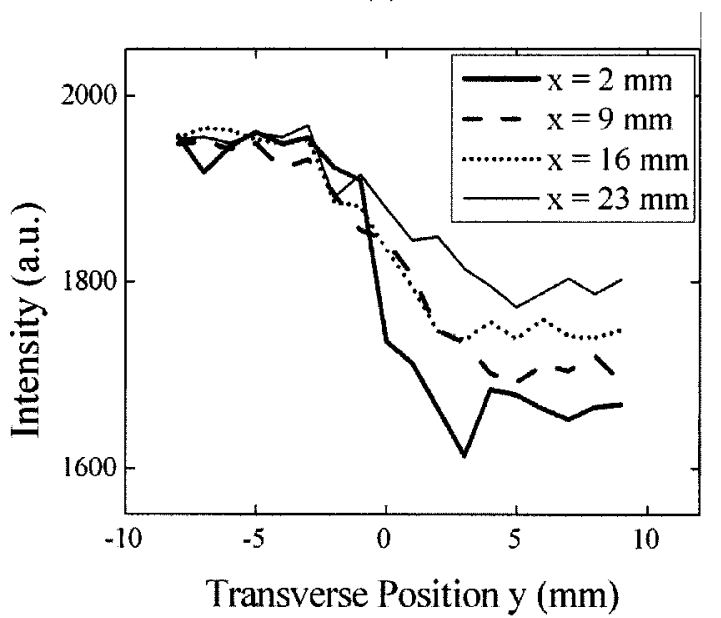

(b)

Fig. 6. Transmitted co-polarized intensity distribution of four target depths obtained from the experiments with blue ink as absorber in the cases (a) without time gating and (b) with time gating.

in Fig. 3(a), the co-polarized intensity shows a strong dependence on the target depth. Again, we calculated the depth indicator $P(x)$ based on the time-gating data in Fig. 3(b). Curve b in Fig. 5 shows the results. It demonstrates better linearity than curve a, indicating the improvement of the accuracy in depth determination with appropriate time gating.

Absorption is another key factor affecting the imaging results in a turbid medium. Although controlling the absorption condition in a turbid medium is usually quite difficult, we conducted an experiment by adding an absorber in the turbid medium to demonstrate the applicability of the proposed target depth determination method in the case of strong absorption. In this experiment, we used blue ink $(0.1 \mathrm{ml})$ as the absorber in the jellied target. The polystyrene microsphere concentrations in the target and background turbid media are the same as those, respectively, in the last experiment leading to the results in Fig. 3. Because the blue ink diffuses from the jellied target into the aqueous background medium and its particle nature creates not only absorption but also extra scattering, it is difficult to estimate the relative levels of scattering and absorption between the target and background media. However, the results of this experiment do show the applicability of the proposed method in the case of stronger absorption.

Figure 6(a) shows the transmitted co-polarized intensity distribution without time gating of four target depths obtained from the experiment with the blue ink as absorber. Compared with the results in Fig. 3(a), one can see data of stronger fluctuation in the case of higher absorption. Figure 6(b) shows the transmitted co-polarized intensity distribution with time gating, corresponding to the results in Fig. 6(a). The time-gating window is the same as that leading to Fig. 3(b). Time gating does smoothen the intensity variation along $y$. Figure 7 shows the depth indicator $P(x)$ in the cases of no time gating (curve a) and time gating (curve b). Although the slopes are relatively smaller than those in Fig. 5 (because of the smaller scattering coefficient contrast between the target and background media), the results here clearly indicate that the proposed target depth determination method is useful in the case of strong absorption. The larger error bars in Fig. 7, as compared with those in Fig. 5, are naturally due to the stronger absorption in the current case. Here again, time gating leads to a $P(x)$ curve of higher linearity.

\section{NUMERICAL MODELING RESULTS}

The experimental results in Section 3 have demonstrated the idea and the feasibility of target depth determination in a turbid medium. As mentioned above, in practical operation, significant medium absorption exists that may result in the degradation of target depth accuracy. It is usually difficult to prepare experimental samples of controlled absorption parameters. To further study the effects of medium absorption and possibly other sample parameters, we developed computer algorithms for Monte Carlo simulations. In this section, we will first demonstrate the consistent results of turbid medium scattering and target depth determination in a case similar to the experimental conditions. The consistency reveals that the developed computer algorithms are appropriate for simulating the turbid scattering systems. The algo-

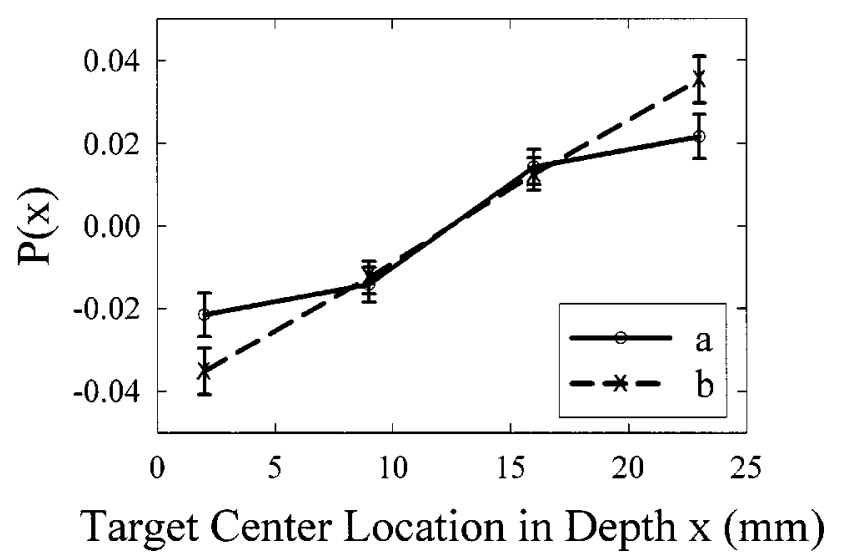

Fig. 7. Depth indicator $P(x)$ versus target depth $x$ in the cases without time gating (curve a) and with time gating (curve b) from the experiments with blue ink as absorber. 


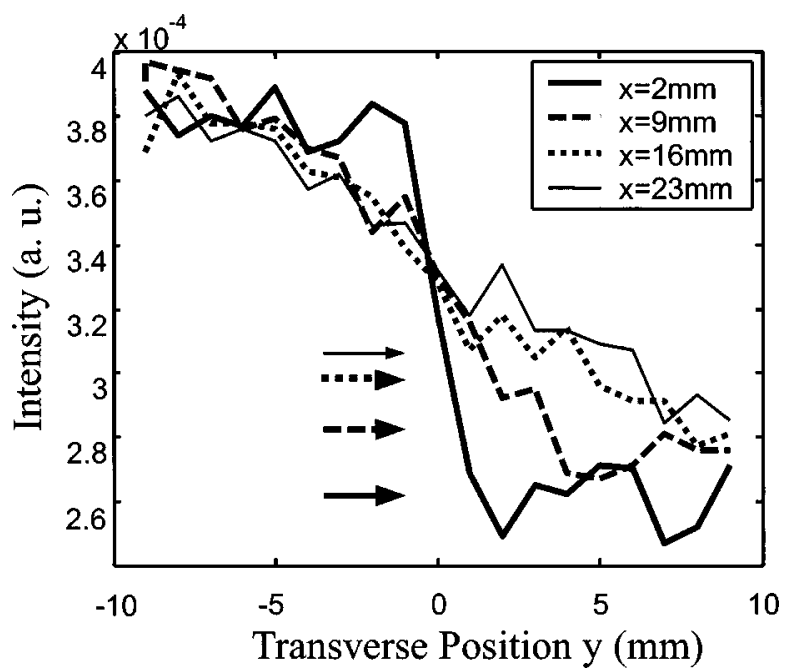

Fig. 8. Transmitted co-polarized intensity distribution with time gating of four target depths obtained from simulations. The scattering coefficients are $\mu_{s}=12.5 \mathrm{~cm}^{-1}$ and $\mu_{s i}$ $=49 \mathrm{~cm}^{-1}$. The absorption coefficient is $\mu_{a}=\mu_{a i}$ $=0.01 \mathrm{~cm}^{-1}$.

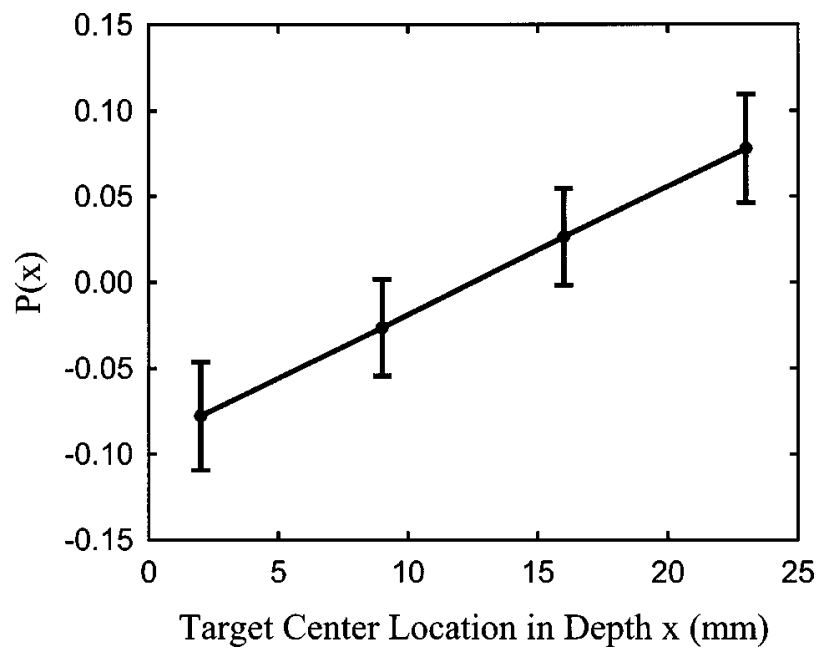

Fig. 9. Depth indicator $P(x)$ versus target depth $x$ from simulations corresponding to the cases in Fig. 8.

rithms are then used for studying the effects of medium absorption on the proposed target depth determination method.

The method of Monte Carlo simulations has been widely discussed. ${ }^{12-14}$ In numerical calculations, the distance between two consecutive scattering events was assumed to be an exponentially distributed random variable, controlled by the extinction coefficient $\mu_{t}$, which is the sum of the scattering coefficient $\mu_{s}$ and the absorption coefficient $\mu_{a}$. The scattering angle was another random variable with its probability density function being determined by the scattering intensity distribution computed with Mie theory. ${ }^{14}$ To include the polarization effect, we evaluated a Stokes vector for each photon. Upon each scattering event, the Stokes vector was multiplied with a rotation matrix and a Mueller matrix. ${ }^{13}$ In other words, coordinate transformation between the local and laboratory coordinate systems was executed at each scattering event. The final Stokes vector can be obtained by properly averaging all the Stokes vectors associated with the received photons. Note that the phase information of signals is neglected in the Monte Carlo simulation. During the random-walk process, we recorded the traversed path length for each contributing photon to allocate the appropriate time instant of the corresponding contribution in the received time-resolved intensity. Note that with our Monte Carlo algorithms, we can compute the timeresolved intensities of different polarizations. In the simulations, the refractive index (1.565) and the diameter $(1.8 \mu \mathrm{m})$ of scattering particles are set the same as those in experiments. The $g$ factor is 0.939421 . It is also assumed that the homogeneous media, which contain microspheres in the background turbid medium and in the target, have the same refractive index of 1.331 .

The results in Figs. 8 and 9 mean to simulate the experimental data in Figs. 3 and 5. The parameters used for simulations are as follows: The scattering coefficients of the background turbid medium and the target object are $\mu_{s}=12.5 \mathrm{~cm}^{-1}$ and $\mu_{s i}=49 \mathrm{~cm}^{-1}$, respectively. The absorption coefficient is $\mu_{a}=\mu_{a i}=0.01 \mathrm{~cm}^{-1}$. All the parameters of scattering geometry (see Fig. 1) are the same as the experimental conditions. For obtaining Figs. 8 and 9, we used 5,000,000 photons for scattering simulations. In Fig. 8, four curves of co-polarized intensity, corresponding to the four target depths, show the strong target depth dependence. The aforementioned shower curtain effect, i.e., the shallower slopes of curves around the target boundary in cases of larger $x$, can also be clearly seen. The arrows indicate the average levels of co-polarized intensity on the target side $(y>0)$. In obtaining these curves, we integrated the time-resolved intensity profile except the tail. In other words, an appropriate time-gating window was chosen for all four cases of different target depths. The tail parts with intensity lower than $5 \%$ of the peaks were truncated. It is noted that the time-gating procedure in this case of numerical simulations is quite different from that in experiments, from which Fig. 3(b) is obtained. In our research, it was found that time gating was an optimization procedure. The gating window may rely on the strengths of scattering and transmitted signals. The buildup of a universal time-gating procedure requires more effort. Figure 9 shows the calculated depth indicator $P(x)$ versus $x$. Here, an almost straight line can be seen, indicating the consistency of simulation results in demonstrating the target depth determination method.

To show the effects of medium absorption, we conducted simulations under the same conditions as those for Figs. 8 and 9, except that the absorption coefficients $\mu_{a}$ and $\mu_{a i}$ are varied. Figures 10 and 11 show the case of varying $\mu_{a}=\mu_{a i}$ from 0.01 to $0.3 \mathrm{~cm}^{-1}$. Figure 10 shows the $y$-dependent co-polarized intensity variations of three $\mu_{a}$ values when the target depth is fixed at $x=2 \mathrm{~mm}$. One can see that the contrast between the target-free and target sides decreases as the absorption coefficient increases (intensity contrasts at $1.15 \times 10^{-4}, 0.95 \times 10^{-4}$, and $0.6 \times 10^{-4}$ for the three cases). The absolute copolarized intensity decreases naturally with higher absorption. From the simulation results, significant target depth dependence of transmitted co-polarized intensity 
can be clearly observed, even with an absorption coefficient as large as $0.3 \mathrm{~cm}^{-1}$. This value represents an overestimate of absorption coefficient in most biological tissues. Figure 11 shows the depth indicators of the three cases of different absorption coefficients. One can see that the depth indicator curve is slightly perturbed as the absorption coefficient increases to $0.3 \mathrm{~cm}^{-1}$. The error bars are longer as the absorption becomes stronger.

To further understand the effects of absorption, we also conducted simulations with $\mu_{a}$ fixed at $0.01 \mathrm{~cm}^{-1}$ but $\mu_{a i}$ varied up to $0.3 \mathrm{~cm}^{-1}$. The scattering coefficients are the same as those leading to the results in Figs. 10 and 11 . Figure 12 shows the $y$-dependent co-polarized intensity variations of three $\mu_{a i}$ values when the target depth is fixed at $x=2 \mathrm{~mm}$. In this situation, although the copolarized intensity levels on the target-free side are approximately the same, those on the target side decrease with increasing $\mu_{a i}$. The contrasts between the target

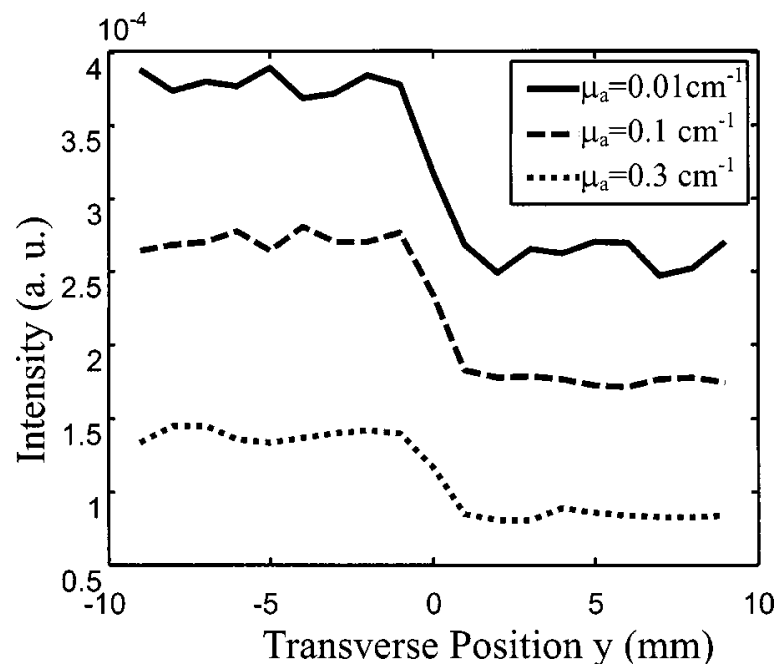

Fig. 10. Transmitted co-polarized intensity distribution with time gating from simulations for three absorption cases: $\mu_{a}$ $=\mu_{a i}=0.01,0.1$, and $0.3 \mathrm{~cm}^{-1}$. The scattering coefficients are fixed at $\mu_{s}=12.5 \mathrm{~cm}^{-1}$ and $\mu_{s i}=49 \mathrm{~cm}^{-1}$, and the target depth is fixed at $x=2 \mathrm{~mm}$.

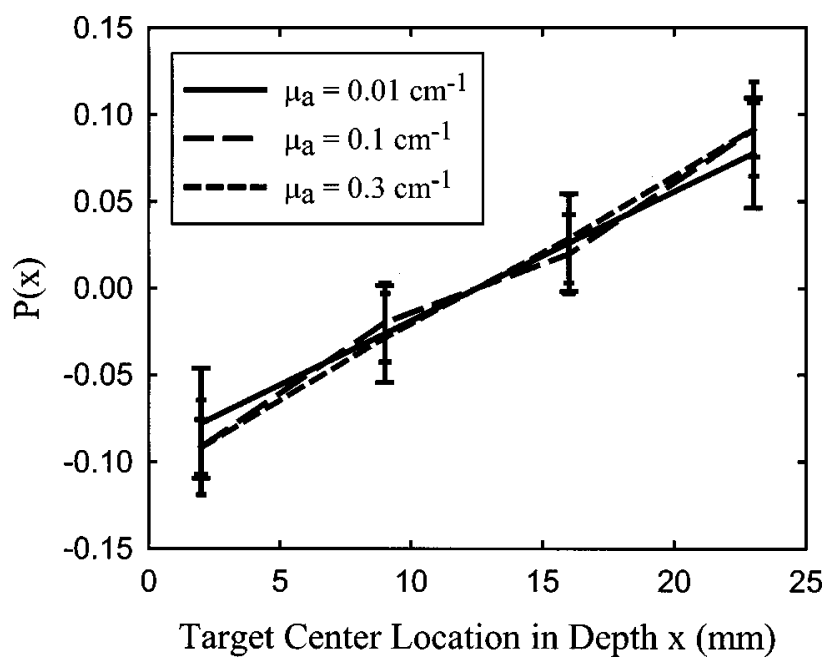

Fig. 11. Depth indicator $P(x)$ versus target depth $x$ from simulations corresponding to the three cases of different absorption coefficients in Fig. 10.

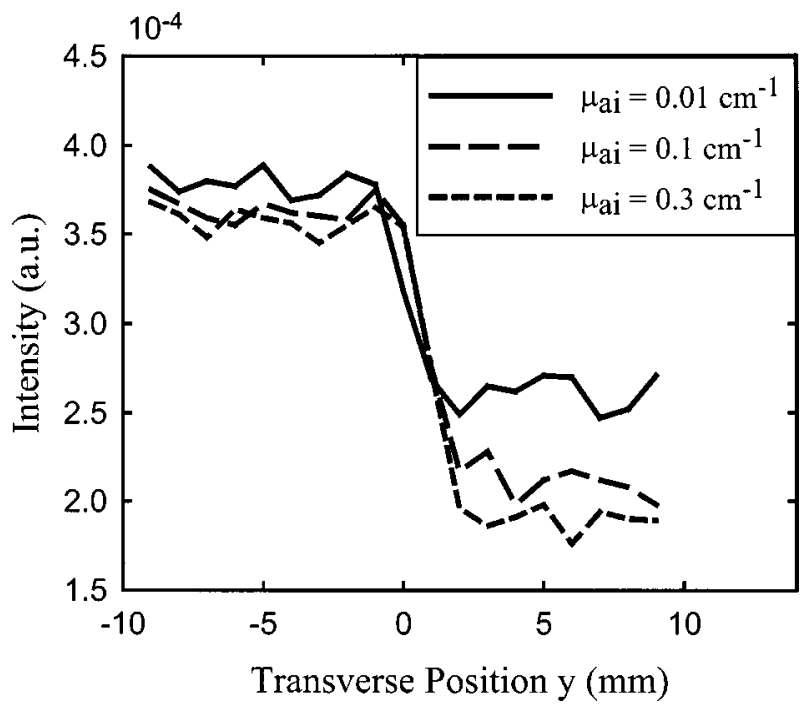

Fig. 12. Transmitted co-polarized intensity distribution with time gating from simulations for three absorption cases: $\mu_{a}$ $=0.01 \mathrm{~cm}^{-1}$ and $\mu_{a i}=0.01,0.1$, and $0.3 \mathrm{~cm}^{-1}$. The scattering coefficients are fixed at $\mu_{s}=12.5 \mathrm{~cm}^{-1}$ and $\mu_{s i}=49 \mathrm{~cm}^{-1}$, and the target depth is fixed at $x=2 \mathrm{~mm}$.

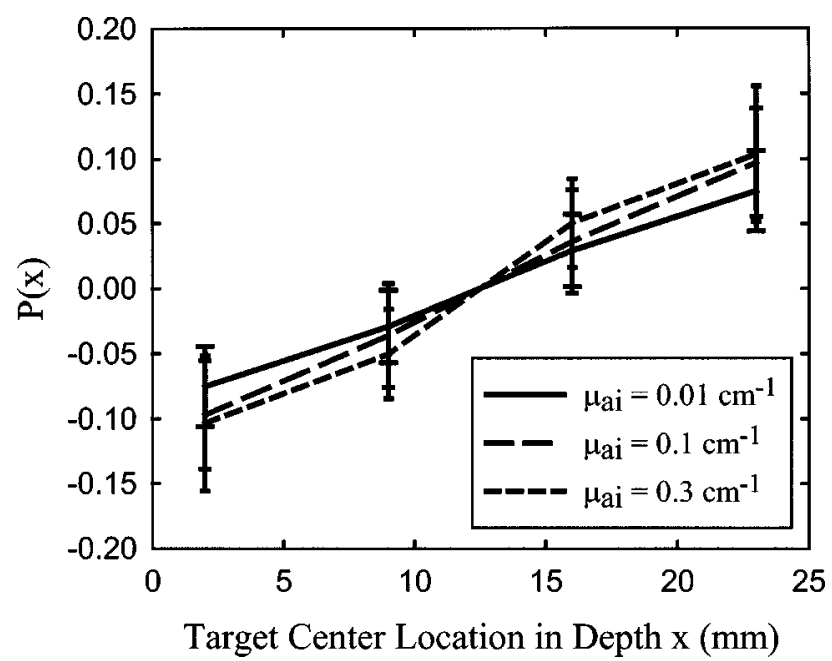

Fig. 13. Depth indicator $P(x)$ versus target depth $x$ from simulations corresponding to the three cases of different absorption coefficients in Fig. 12.

and target-free sides are $1.2 \times 10^{-4}, 1.5 \times 10^{-4}$, and $1.7 \times 10^{-4}$, respectively, for the cases of $\mu_{a i}=0.01,0.1$, and $0.3 \mathrm{~cm}^{-1}$. Figure 13 shows the depth indicators of the three cases. One can see that the slope of $P(x)$ increases slightly with increasing $\mu_{a i}$. This result can be attributed to the increasing contrast of optical properties (including scattering and absorption) between the target and background media. Such a result, together with those in Figs. 5, 7, 9, and 11, indicates that the proposed target depth determination method is applicable to random scattering systems with quite large ranges of scattering coefficient and absorption coefficient.

\section{CONCLUSIONS}

In summary, we have experimentally demonstrated a novel method for target depth determination in a turbid 
medium. This method relies on the strong dependence of transmitted co-polarized intensity on target depth. Such a dependence originates from the inclusion of certain diffuse photons in the co-polarized intensity. A target of stronger scattering located closer to the transmitter results in stronger photon divergence and hence weaker copolarized intensity at the receiver of a finite aperture. On the other hand, the DOP carries only information about ballistic and snake photons. It is weakly dependent on the target depth. DOP data can be used as a reference of absolute scattering strength in the turbid system. They can also be used for constructing high-quality two-dimensional images. ${ }^{15}$ Our experimental and Monte Carlo simulation results have shown the feasibility of the proposed target depth determination method. Meanwhile, an appropriate time-gating process can help in improving the accuracy of target depth. In addition, the results show that the proposed method has quite large applicable ranges of scattering coefficient and absorption coefficient.

\section{ACKNOWLEDGMENT}

This research was supported by National Health Research Institute, Republic of China, under grant NHRIEX92-9220EI.

Address correspondence to C. C. Yang, Graduate Institute of Electro-Optical Engineering, National Taiwan University, No. 1, Section 4, Roosevelt Road, Taipei, Taiwan; phone, 886-2-23657624; fax, 886-2-23652637; e-mail, ccy@cc.ee.ntu.edu.tw.

\section{REFERENCES}

1. R. R. Alfano, X. Liang, L. Wang, and P. P. Ho, "Timeresolved imaging of translucent droplets in highly scattering turbid media," Science 264, 1913-1915 (1994).

2. D. Grosenick, H. Wabnitz, H. H. Rinneberg, K. T. Moesta, and P. M. Schlag, "Development of a time-domain optical mammography and first in vivo applications," Appl. Opt. 38, 2927-2943 (1999).

3. S. K. Gayen, M. Alrubaiee, H. E. Savage, S. P. Schantz, and R. R. Alfano, "Parotid gland tissues investigated by picosecond time-gated and optical spectroscopic imaging techniques," IEEE J. Sel. Top. Quantum Electron. 7, 906-911 (2001).

4. X. Gan, S. P. Schilders, and Min Gu, "Image enhancement through turbid media under a microscope by use of polarization gating methods," J. Opt. Soc. Am. A 16, 2177-2184 (1999).

5. V. Sankaran, K. Schönenberger, J. T. Walsh, Jr., and D. J. Maitland, "Polarization discrimination of coherently propagating light in turbid media," Appl. Opt. 38, 4252-4261 (1999).

6. Chia-Wei Sun, Long-Sheng Lu, C. C. Yang, Yean-Woei Kiang, and Ming-Jai Su, "Myocardial tissue characterization based on the time-resolved Stokes-Mueller formalism," Opt. Express 10, 1347-1353 (2002), www.opticsexpress.org.

7. U. Hampel, E. Schleicher, and R. Freyer, "Volume image reconstruction for diffuse optical tomography," Appl. Opt. 41, 3816-3826 (2002).

8. F. Gao, P. Poulet, and Y. Yamada, "Simultaneous mapping of absorption and scattering coefficients from a threedimensional model of time-resolved optical tomography," Appl. Opt. 39, 5898-5910 (2000).

9. M. E. Kilmer, E. L. Miller, D. Boas, and D. Brooks, "A shape-based reconstruction technique for DPDW data," Opt. Express 7, 481-491 (2000), www.opticsexpress.org.

10. H. H. Wang, C. W. Sun, Y. M. Wang, Y. W. Kiang, and C. C. Yang, "Determination of the depth of a scattering target in a turbid medium with polarization discrimination of transmitted signals," Opt. Lett. 28, 25-27 (2003).

11. I. Dror, A. Sandrov, and N. S. Kopeika, "Experimental investigation of the influence of the relative position of the scattering layer on image quality: the shower curtain effect," Appl. Opt. 37, 6495-6499 (1998).

12. X. Wang and L. V. Wang, "Propagation of polarized light in birefringent turbid media: time-resolved simulations," Opt. Express 9, 254-259 (2001), www.opticsexpress.org.

13. S. Bartel and A. H. Hielscher, "Monte Carlo simulations of the diffuse backscattering Mueller matrix for highly scattering media," Appl. Opt. 39, 1580-1588 (2000).

14. L. Wang, S. L. Jacques, and L. Zheng, "MCML-Monte Carlo modeling of light transport in multi-layered tissues," Comput. Methods Programs Biomed. 47, 131-146 (1995).

15. C. W. Sun, C. C. Yang, and Y. W. Kiang, "Optical imaging based on time-resolved Stokes vector in filamentous tissues," Appl. Opt. 42, 750-754 (2003). 